\section{Role of clinical informatics in the implementation of universal health coverage}

To the Editor: On 23 September 2019, the United Nations General Assembly held a high-level meeting on universal health coverage (UHC). This meeting, held under the theme 'UHC: Moving together to build a healthier world, aims to accelerate progress toward UHC, including financial risk protection, access to quality essential healthcare services and access to safe, effective, quality and affordable essential medicines and vaccines for all. ${ }^{[1]}$ E-health (or health/clinical informatics) is recognised as one of the key components of successful implementation of UHC by the World Health Organization, which states that 'It has become increasingly clear that UHC cannot be achieved without the support of eHealth, ${ }^{[2]}$ which was also acknowledged by the World Health Assembly: 'eHealth is the cost-effective and secure use of ICT [information and communications technology] in support of health and health-related fields, including health-care services, health surveillance, health literature, health education, knowledge and research. ${ }^{[3]}$

In South Africa (SA), strategic issues that highlighted the 2012 2017 e-health strategy ${ }^{[4]}$ were as follows: widely differing levels of e-health maturity across and in provinces; a large number of disparate systems with little or no interoperability and communication; several past initiatives that have not reached fruition because of poor planning or lack of consistent sponsorship, management and/or funding; and a low degree of co-operation, collaboration and sharing across all sectors. Reasonable progress has since been made in certain areas, such as the developing new e-health strategy, the National Health Normative Standards Framework for interoperability, the Health Patient Registration System, and the provision of e-health infrastructure ${ }^{[5]}$ However, the massive investments associated with this progress have not been met with corresponding investments in capacity building of healthcare personnel, who are the ultimate users of the deployed systems. They not only generate data through e-health, but also use these data for effective and efficient decisionmaking - even at grass-roots level. It is in this light that newly designed training programmes in clinical informatics would be necessary, covering broad concepts in an electronic health record (EHR) system, its implementation, as well as its adoption for decision-making, especially in the clinical setting.

Clinical informatics includes the study and practice of an information-based approach to healthcare delivery in which data must be structured in a certain way to be effectively retrieved and used for decision-making. ${ }^{[6]}$ The EHR system forms the backbone of clinical informatics. All of this information must be collected, stored, interpreted, analysed and implemented into a treatment plan. Training in clinical informatics should integrate computer science, clinical data and application of information technology in healthcare settings. It would assist health professionals to process information and to manage patient populations routinely, thus bridging the gap between personal and population health that has existed for more than a century.

It is important for SA universities to partner with public and private sector institutions to develop integrated training programmes

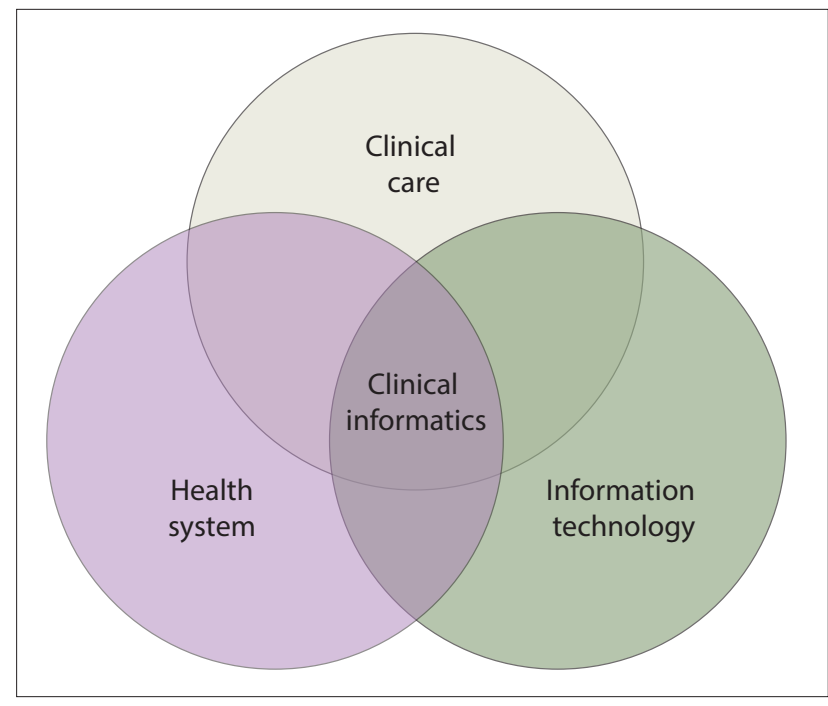

Fig. 1. Concept of clinical informatics.

for healthcare professionals, if SA wishes to achieve its objectives associated with UHC. In view of this, the University of Pretoria has decided to develop formal continuous professional development programmes on clinical informatics, which would be available from 2020.

\section{T C R Maimela}

Department of Public Health Medicine, Steve Biko Academic Hospital and University of Pretoria; and University of Pretoria COPC Research Unit, Pretoria, South Africa

tshegomaimela@gmail.com

\section{N Mutshekwane}

Charlotte Maxeke Research Cluster, and MEDITECH, Johannesburg, South Africa

\section{Basu}

Department of Public Health Medicine, Steve Biko Academic Hospital and University of Pretoria; and University of Pretoria COPC Research Unit, Pretoria, South Africa

\footnotetext{
1. General Assembly, United Nations. Universal health coverage. 2019. https://www.un.org/pga/73/ event/universal-health-coverage/ (accessed 30 September 2019).

2. World Health Organization. AFRO. Global diffusion of eHealth: Making universal health coverag achievable. 2016. https://www.afro.who.int/publications/global-diffusion-ehealth-making-universalhealth-coverage-achievable (accessed 30 September 2019).

3. World Health Organization. WHO eHealth Resolution WHA58.28. 2005. https://www.who.int healthacademy/news/en/ (accessed 30 September 2019).

National Department of Health. National eHealth Strategy, South Africa 2012 - 2017. 2012. https://ndoh. dhmis.org/owncloud/index.php/s/R5 cmdp0gY4Fa43Z?path=/eHealth (accessed 30 September 2019). National Department of Health. National Health Normative Standards Framework for Interoperability National Department of Health. National Health Normative St
in eHealth in South Africa. Version 2.0. Pretoria: $\mathrm{NDoH}, 2014$.

6. Duke University. Clinical informatics. https://mmci.duke.edu/about-mmci/clinical-informatics (accessed 30 September 2019)
}

S Afr Med J 2019;109(12):898. https://doi.org/10.7196/SAMJ.2019.v109i12.14445 